\title{
Las personas migrantes desaparecidas y sus familiares ${ }^{1}$
}

\author{
Missing migrants and their families
}

Comité Internacional de la Cruz Roja $(\mathrm{CICR})^{2}$

www.cicr.org

Recibido: 24/09/2015 - Aceptado: 11/11/2015

\section{Resumen}

Los familiares de personas migrantes desaparecidas sufren distintas afectaciones en su vida a raíz de la desaparición de su ser querido. Por encima de todo, necesitan que se busque a su familiar, averiguar dónde se encuentra y qué le ha ocurrido. Esto se manifiesta claramente en sus prioridades, a las que se suman otras necesidades derivadas de la desaparición de carácter económico, psicológico, legal, administrativo y de reconocimiento de su situación.

Debido a la ausencia de rutas de atención claras y procedimientos sistemáticos por parte de las autoridades, existe una gran atomización en las prácticas de búsqueda. Las familias se ven obligadas a contactar distintas instancias, e incluso a repetir trámites — presentando nuevamente la misma información y documentaciónante la misma autoridad. Lo anterior ocasiona un alto riesgo de re-victimización para las familias, así como el incremento de los gastos generados por la búsqueda.

El Comité Internacional de la Cruz Roja (CICR) considera que el fenómeno de la desaparición de personas migrantes trasciende las fronteras de los países implicados, por lo que propone un enfoque regional para abordarlo. Las

\section{Abstract}

The relatives of missing migrants suffer from different consequences in their lives as a result of the disappearance of a loved one. Above all, they need their relative to be searched for; they need to find out where he or she is and what happened to him or her. This can be clearly seen in their priorities along with the other needs derived from the economic, psychological, legal, and administrative disappearance, as well as the lack of acknowledgement of their situation.

There is a great fragmentation in the search practices due to the lack of clear attention and systematic procedures from the authorities.

Families are forced to approach different institutions and to repeat procedures before the same authorities-thus submitting the same information and documentation all over again--. This causes a high risk of re-victimization for the families as well as a raise in the expenses generated by the search.

The International Committee of the Red Cross (ICRC) considers that the phenomenon of the disappearance of migrants goes beyond the borderlines of the countries

1 El presente artículo es un extracto de la publicación del CICR: “Evaluación sobre la situación de las familias de personas migrantes desaparecidas. El Salvador, Guatemala, Honduras y México", 2014.

2 El Comité Internacional de la Cruz Roja (CICR) fundado en 1863, trabaja en todo el mundo para prestar ayuda humanitaria a las personas afectadas por los conflictos y la violencia armada, y para promover las leyes por las que se protege a las víctimas de la guerra. Es una Institución independiente y neutral, su cometido dimana esencialmente de los Convenios de Ginebra de 1949. 
autoridades deben no solo tomar medidas en cada uno de los ámbitos nacionales, sino también coordinarlas a escala regional.

\section{Palabras clave}

Migración, ayuda internacional, población desaparecida. in question; therefore, it suggests a regional approach to tackle it. The authorities have to not only take measures in each of the national environments, but also to coordinate them at the regional scale.

\section{Keywords}

Migration, international aid, missing people.
Evaluación sobre la situación de las familias de personas migrantes desaparecidas

Entre febrero y julio de 2013, el CICR llevó a cabo una evaluación regional sobre la situación de las familias de personas migrantes desaparecidas en tránsito hacia los Estados Unidos. Se entrevistó a 218 familias, de las cuales 44 se hicieron en El Salvador, 62 en Guatemala, 68 en Honduras y 44 en México. Adicionalmente se llevaron a cabo 48 entrevistas a instituciones del Estado y organizaciones de la sociedad civil implicadas en la temática de las personas migrantes desaparecidas.

Se recopiló información fundamentalmente acerca del perfil de la persona entrevistada y de su hogar, sus prioridades, las principales dificultades relacionadas con los ámbitos de la búsqueda, incluyendo aspectos administrativos, legales, socioeconómicos, psicosociales y forenses, de sus mecanismos de afrontamiento, así como de aquello que solicitan a las autoridades.

\section{Principales resultados}

\section{La necesidad de saber: búsqueda y obtención de información}

La gran mayoría de los entrevistados manifestaron necesitar, por encima de todo, información acerca de sus seres queridos y estar seguros de que se realizaron todas las acciones necesarias para localizarlos. Esta es su prioridad absoluta, a pesar de que indicaron tener, como consecuencia de la desaparición de su familiar, otros problemas que se tratarán más adelante en este documento.

\section{La desaparición}

Con relación a las razones de la desaparición, la mayoría de los familiares consideran un escenario amplio y complejo: imaginan a sus seres queridos en situación de privación de libertad, ingresados en hospitales, víctimas de trata de personas o trata de órganos, o reclutados forzadamente por actores de la violencia armada, en particular, en el ámbito del tráfico de drogas. Cerca de una cuarta parte de los entrevistados $(23,9 \%)$ cree que su familiar sufrió alguna forma de violencia relacionada con su desaparición (amenazas, secuestros, extorsiones, etc.).

Cabe mencionar que algunas personas migrantes no volvieron a comunicarse con sus familias desde el momento de su salida, de manera que el último lugar de contacto coincide con su comunidad de origen. Esto refleja la necesidad de generar programas de información acerca de los riesgos de la ruta y la importancia de mantener el contacto entre las personas migrantes y sus familias.

\section{La búsqueda}

Las familias entrevistadas se dirigieron a una gran variedad de fuentes de información para buscar a sus seres queridos, principalmente a instituciones estatales y a organizaciones de la sociedad civil y, en menor medida, a otros actores, tales como familiares residentes en otro país, polleros y coyotes, líderes religiosos, abogados, videntes y medios de comunicación.

En muchas ocasiones se vieron obligados a hacer la misma denuncia dos o tres veces ante la misma autoridad, o bien tuvieron que presentar sus casos en distintas entidades. Esto conlleva a entregar una vez más todos los detalles de la desaparición y los documentos requeridos, acumulando gastos para los familiares (tiempo, transporte, copias, fotos, etc.) y, peor aún, reviviendo el dolor ocasionado por la desaparición del ser querido y corriendo el riesgo de revictimización cada vez que acuden a una nueva entrevista. Son muy pocos los familiares que recibieron una llamada de seguimiento por parte de las autoridades que los atendieron, 
a pesar de que les aseguraron que se comunicarían con ellos. como resultado, las personas entrevistadas expresaron haber perdido la confianza en las autoridades y esto, unido a la falta de resultados, explicaba que se hubieran visto obligadas a emprender la búsqueda por sus propios medios. Estas familias expresaron que prefieren que se las mantenga informadas, aunque no exista ningún avance, a quedarse en la incertidumbre acerca de lo que está sucediendo.

\section{Coordinación regional}

Existe un amplio abanico de instituciones que reciben casos de personas migrantes desaparecidas, sin embargo, pocas son las que realizan una búsqueda activa de forma sistemática. La acción emprendida por numerosas instancias que reciben la solicitud consiste en canalizar los casos a otras, a menudo mediante oficios que contienen información insuficiente para un proceso de búsqueda, localización e identificación. La comunicación y coordinación entre actores a escalas local y regional no está estructurada.

No está expresamente delimitada la responsabilidad de cada instancia a lo largo del proceso de búsqueda: la que recibe la información sobre el caso, la que tramita y efectúa la búsqueda, la que administra los datos y la que emite la información al familiar. En la actualidad, las familias se adentran en un laberinto institucional en el que dependen en gran medida de la buena voluntad y disposición de los funcionarios que las atienden.

Otro de los principales problemas identificados es la ausencia, a escala regional, de un registro unificado consolidado y depurado de personas desaparecidas, así como de un adecuado registro de personas fallecidas sin identificar y de registros completos de internos en centros de detención, hospitales y otros lugares donde se podría localizar a la persona desaparecida en vida.

\section{Recomendaciones}

- Establecer claramente las competencias de cada institución implicada en todos los aspectos relacionados con la problemática de personas migrantes desaparecidas, y especificar qué instituciones funcionarán como puntos de atención únicos para familiares y de registro de casos de personas desaparecidas; por ejemplo, ministerios de relaciones exteriores, procuradurías o fiscalías, instituciones forenses, oficinas de derechos humanos, etc.
Explicar las distintas competencias de cada institución en materia de personas migrantes desaparecidas de una manera clara y transparente a los familiares. Esta información se puede difundir por medio de campañas de comunicación masiva (internet, radio, televisión, anuncios en transporte público, etc.).

- Cada punto de atención deberá contar con espacios adecuados que garanticen la privacidad durante la entrevista con la familia, y con recursos humanos sensibilizados y capacitados para la correcta toma de información. Esto contribuirá a la creación de un clima de confianza y dignificación de la atención a la familia.

- Definir claramente la institución que se hará cargo de darle seguimiento a cada caso a lo largo de todo el proceso de búsqueda, y que sea esta la responsable de mantener informada a la familia sobre las acciones, procesos y resultados, a través de una comunicación regular.

- Contar con formularios de entrevista homologados y una base de datos que facilite la sistematización y centralización de los datos obtenidos, que simplifique el intercambio de información entre los puntos de atención, así como el seguimiento de los casos individuales.

- La gestión de la información deberá siempre contemplar las reglas básicas de protección de datos, que incluye la explicación de manera clara, a los familiares y a otras fuentes de información, sobre la finalidad y el uso que se le dará a la información recabada, las instancias con las que será compartida y la autoridad que estará encargada de proteger y custodiar dicha información.

\section{Necesidades relacionadas con el ámbito forense}

La mayoría de las familias entrevistadas creen que su ser querido está vivo. Para ellas, en caso de que se confirme el fallecimiento de su ser querido desaparecido, resulta fundamental que les proporcionen información confiable y certera acerca de la identificación, y que no les entreguen los cuerpos incinerados. Consideran necesario recuperar el cuerpo para darle sepultura y visitar el lugar de entierro regularmente.

Teniendo en cuenta la información proporcionada por las instituciones entrevistadas, se observó con preocupación que las probabilidades de identificar a un migrante fallecido, en cualquier punto de la ruta migratoria, son remotas. Los procesos de identificación forense no están sistematizados ni estandarizados. 
Es importante resaltar, además, que muchos fallecidos sin identificar se encuentran inhumados en fosas comunes. En los casos en que se inhuman individualmente, no hay un registro de cementerio que permita su posterior recuperación. Asimismo, el registro post mortem (perfil biológico, fotos, huellas dactilares, formato post mortem, muestra para análisis genético posterior y registro dental) no es aplicado en todos los cadáveres.

Adicionalmente, se ha podido constatar en los cuatro países objeto de esta evaluación que las instituciones forenses carecen de equipos humanos especializados en la comparación de datos, lo que dificulta la posterior identificación de personas fallecidas que son declaradas no identificadas. También carecen de oficinas de atención a los familiares.

Cuestiones adicionales como la ausencia de comunicación entre instituciones, la escasa calidad del trabajo técnico forense y el presupuesto asignado a los servicios médicos forenses, claramente insuficiente, repercuten negativamente en la satisfacción de las necesidades de información a los familiares.

\section{Recomendaciones}

- Establecer un mandato definido para la atención de casos por parte de las instituciones forenses. Estas deberán desarrollar una estrategia para la atención de las familias, con el fin de responder a sus necesidades de información y de toma de decisiones en las distintas etapas del proceso de recolección de datos ante mortem, recuperación, análisis e identificación forense.

- Se deberá hacer partícipe a las familias o a sus representantes legales (ministerios de relaciones exteriores) en todo el proceso; desde la recopilación de información ante mortem, la presentación de información a las familias que resulte de la búsqueda en los registros de personas fallecidas, entrega y explicación del informe de resultados, hasta la recuperación de los restos, en su caso.

- Las autoridades forenses deberán informar a los familiares de manera clara y sencilla acerca de los diferentes métodos de identificación, sus alcances y sus límites.

- Los ministerios/secretarías de relaciones exteriores e instituciones forenses de todos los países de la región, deberán fortalecer las competencias técnicas de sus equipos y promover la cooperación regional para la cooperación e intercambio de información, la búsqueda de personas desaparecidas, la recuperación de restos humanos y su identificación y restitución, utilizando las disciplinas de la ciencia forense que sean pertinentes y necesarias en cada caso.

- Para facilitar la localización e identificación de personas migrantes fallecidas, resulta importante el desarrollo de registros dactilares, médicos y odontológicos en los países de origen de la población migrante.

- Con la finalidad de erradicar la práctica de inhumación de cadáveres sin identificar en fosas comunes, así como la incineración de los cadáveres antes de la devolución a sus familiares, se recomienda que:

- Se efectúe la reforma de las leyes (si existen) que permiten estas prácticas y se establezca su prohibición legal, en los cuatro países.

- Las autoridades encargadas de la búsqueda de personas y aquellas de las que dependan los servicios forenses, deberán adoptar medidas para prohibir estas prácticas, comenzando por su normativa interna y sus protocolos de actuación, ya sea a través de la creación o de la adecuación de estos.

\section{Necesidades en el ámbito psicológico, psicosomático y psicosocial}

El sufrimiento de las familias con un ser querido desaparecido no tiene límite en el tiempo, se perpetúa hasta que los familiares reciben noticias sobre la suerte y el paradero de su allegado. Este dolor conlleva una serie de consecuencias graves a niveles psicológico, psicosomático y psicosocial.

- Consecuencias psicológicas de la desaparición:

Para los familiares, al no contar con información verídica sobre lo ocurrido con su ser querido ni existir evidencia de la muerte, se suspende todo proceso normal de duelo. Tampoco hay reconocimiento social de la pérdida, ni rituales sociales que apoyen a la familia para enfrentar el dolor.

- Las personas entrevistadas parecen haber detenido su vida a partir de la desaparición y viven esperando que el desaparecido regrese.

El miedo expresado por los familiares se relaciona con la probabilidad de que su familiar haya fallecido, a perder al ser amado y a la fantasía de lo que pudo ocurrirle, provocando una intensa angustia. Asimismo, expresaron miedo a que se supiera su historia, a ser 
extorsionados por los coyotes y por los actores de la violencia armada, o porque conocían el contexto en el que se produjo la desaparición.

- El sentimiento de tristeza y depresión se manifestó en el $98,2 \%$ de los entrevistados. Para la mayoría de ellos, la vida ya no tiene sentido con un familiar ausente.

- Las familias se responsabilizan a ellas mismas por no haber evitado que su familiar migrara, o por no haberlo podido proteger. En la comunidad, consideran a los más cercanos como responsables y en algunos casos los estigmatizan. Las mujeres muchas veces se llevan la peor parte, pues los vecinos desconfían de ellas y reciben duras críticas de sus propios familiares por no haber podido cuidar de los suyos, o por querer tener mejores condiciones económicas.

\section{- Consecuencias psicosomáticas derivadas} de la desaparición:

La mayoría de las personas expresaron sentirse enfermas desde que desapareció su familiar. Entre las consecuencias más mencionadas se encuentran las siguientes: sentir palpitaciones y problemas para respirar, en un $59,4 \%$ de los entrevistados; dolores de cabeza, que alcanzan a un 79,2 \%; y dolor de corazón, para el $62 \%$.

- Consecuencias psicosociales originadas por la desaparición:

En la evaluación se analizaron también las consecuencias de la desaparición desde un enfoque psicosocial, el cual reconoce al ser humano desde una perspectiva integral y sistémica, es decir, inmerso en un medio familiar, comunitario, social, cultural y político, del que es indisociable.

\section{- La persona desaparecida y la comunidad}

La desaparición es un fenómeno conocido en Guatemala, El Salvador, Honduras y México, por lo que ya se cuenta con memorias similares. Sin embargo, la respuesta colectiva en el pasado fue distinta: se formaron organizaciones de víctimas para buscar a los desaparecidos, crearon un sistema de apoyo mutuo, de denuncia y acompañamiento. No obstante, ante la situación específica de las personas migrantes desaparecidas, el apoyo social es mucho menor, la organización social es incipiente y la problemática no está suficientemente visibilizada.

\section{La familia del desaparecido}

A las personas entrevistadas, además de sufrir la pérdida del ser querido, se les alteró el sistema familiar: mencionaron separaciones y aumento de la responsabilidad hacia los que quedaron. Ante la desaparición son necesarios reacomodos internos $y$, generalmente, las abuelas, abuelos o tías asumieron el cuidado de los niños huérfanos, mientras tanto.

A la desaparición del ser amado se suman otras pérdidas para las familias: de valores, de confianza en el Estado y en los otros, o del proyecto de vida. La desaparición ha minado el sistema de creencias que le da sentido a la vida, llevando a las personas a un estado de crisis existencial.

Los niños viven la ausencia igual que el resto del sistema familiar, aunque sin contar con algunos elementos necesarios para comprender lo que sucede. También sufren cambios en su ambiente habitual, desintegración familiar, tienen que dejar su vivienda para ubicarse en casas de familiares, lo que conlleva a la ruptura de los referentes más inmediatos. Debido a la falta de recursos, algunos niños tienen que abandonar la escuela y dedicarse a trabajar.

Las mujeres expresaron que la vida dentro del hogar cambió radicalmente desde que desapareció su ser amado. En la mayoría de los casos, el familiar desaparecido era el principal sustento del hogar, de modo que en poco tiempo las mujeres se vieron enfrentadas a asumir nuevos papeles, se responsabilizaron casi exclusivamente del cuidado de los otros y asumieron el deber de garantizar el sustento económico de los demás miembros.

\section{Recomendaciones}

Las instituciones responsables de atender a las familias deben contar con funcionarios sensibilizados y equipos multidisciplinarios que sean capaces de asegurar una atención integral con enfoque psicosocial. Esto incluye la detección y el fortalecimiento de redes, tanto 
institucionales como de la sociedad civil, a las cuales los familiares puedan acudir.

- Los funcionarios encargados de atender a las familias deben conocer y comprender el impacto psicosocial y psicológico de la desaparición, con el fin de brindar una atención diferencial y pertinente a las características y necesidades de las familias.

- Es esencial que se considere el impacto emocional que generan los casos sobre los funcionarios que les dan seguimiento, y garantizar espacios de cuidado para ellos, facilidades para recibir terapia, rotación del personal para evitar pasar demasiado tiempo en el mismo puesto, actividades recreativas, etc.

- Fomentar espacios de encuentro con las familias y entre ellas mismas, que funcionen como grupos de reflexión, apoyo e información, así como de reconocimiento a su situación.

- Tomar en cuenta los aspectos significativos referentes a la cultura, género y edad de la población a la que se atenderá.

\section{Necesidades económicas}

Los principales problemas económicos para los familiares se deben a la disminución de sus ingresos, ya que en la mayoría de los casos la persona ausente era el principal sustento o aportaba importantes ingresos al hogar. Los familiares deben hacer frente a una mayor responsabilidad económica al verse forzados a responsabilizarse de los hijos o de la familia entera de la persona desaparecida. Igualmente, hay gastos adicionales generados por la búsqueda: desembolsos administrativos y de transporte para poder acudir a organizaciones de la sociedad civil o a instituciones del Estado, que quedan muy alejadas de sus lugares de origen. Lo anterior se refleja en el hecho de que el $49,8 \%$ de los entrevistados manifiesta haber tenido problemas para llegar a fin de mes, y que el $36 \%$ de ellos depende ahora de la ayuda de terceros.

\section{Recomendaciones}

- Evitar que la búsqueda genere gastos adicionales a la familia. Esto se puede lograr a través de una ruta de atención definida y eficiente que las familias conozcan (ver recomendaciones de búsqueda).

- Considerar a los familiares de personas migrantes desaparecidas como sujetos de atención, facilitar su acceso a los programas sociales que ofrece el Estado (económicos, de vivienda, salud, formación profesional, becas para menores, etc.), especialmente con un enfoque productivo, que promueva el desarrollo o la mejora de los medios de subsistencia de las familias.

- Reconocer a los familiares de personas desaparecidas jurídicamente como víctimas y considerar su situación económica derivada de la desaparición para permitirles la negociación de los plazos de pago de las deudas contraídas con instituciones bancarias, de crédito, hipotecarias, etc.

\section{Obstáculos en materia legal y administrativa}

En términos generales, la mayor parte de los entrevistados manifestaron no enfrentar problemas legales como consecuencia de la desaparición de su familiar. Un análisis preliminar de estos datos puede apuntar a que las familias no contemplan la posibilidad de que exista un sistema de protección estatal que las considere como sujetos de derechos, a diferencia de muchos familiares de desaparecidos a raíz de conflictos armados que sí cuentan con esta protección e información.

Quienes mencionaron haber tenido dificultades de este tipo se refirieron principalmente a problemas relacionados con la disposición del patrimonio y con obligaciones contraídas por la persona desaparecida. Algunas familias mencionaron que, debido al coste económico que implica el viaje, solicitaron préstamos - con altos intereses- que pagarían una vez que la persona Ilegara a Estados Unidos. La garantía de los créditos a menudo consiste en las propiedades que las familias pierden al no poder afrontar la deuda.

El $13 \%$ explicó haber tenido dificultades con la disposición de propiedades, principalmente de la vivienda familiar, que figuraba registrada a nombre de la persona desaparecida. Algunos familiares tuvieron problemas para el pago de deudas de las personas desaparecidas, teniendo incluso que hipotecar la vivienda. El resto de los entrevistados mencionó dificultades para obtener documentos (13,2 \%), lograr la custodia de los hijos (12,3 $\%)$ y acceder a cuentas bancarias (5,1\%).

Cabe notar que en todos los países fueron muy pocos los entrevistados que mencionaron problemas relacionados con el trámite de pensiones y el acceso a cuentas bancarias, lo cual podría estar relacionado con el hecho de que la mayoría de las familias no están cubier tas por un sistema de seguridad 
social, sus integrantes se encuentran desempleados 0 activos en el mercado de trabajo informal y que su acceso a los servicios financieros básicos son muy limitados.

Los códigos civiles de los cuatro países contemplan figuras de declaratoria de ausencia y presunción de muerte. Sin embargo, limitan quiénes pueden interponer la acción, y su tramitación suele ser costosa y demorada, al requerir de un cierto número de años (entre dos y tres) para interponer la solicitud, como de la publicación de edictos o anuncios por cuenta del promovente, entre otros gastos, que desincentivan a los familiares a optar por esta vía. Por otra parte, los efectos de la declaración de ausencia consisten en nombrar administradores de bienes, pero no tiene ningún impacto sobre temas de reconocimiento de la personalidad jurídica, seguridad social para los familiares o patria potestad.

Finalmente, para que los herederos tengan la posesión definitiva de los bienes deben solicitar que el juez civil declare la presunción de muerte de la persona desaparecida después de seis años (aproximadamente) de que se declaró la ausencia. Esta opción plantea una serie de dilemas para los familiares, ya que deben solicitar que se presuma la muerte de la persona sin que esta haya sido encontrada 0 sin que se aclaren las circunstancias de la desaparición.

\section{Recomendaciones}

- Garantizar que los familiares de personas desaparecidas en cada país puedan interponer una acción sencilla que permita otorgar un estatuto jurídico específico a su situación, y que este permita a los familiares agilizar trámites relativos a la seguridad social, propiedades y negociación de créditos y deudas, entre otras necesidades. La declaración de ausencia por desaparición o declaración de desaparición debe tener plazos y efectos aceptables para los familiares.

- Informar de manera clara, sencilla y oportuna a los familiares sobre la normativa, procedimientos, mecanismos y procesos legales existentes para esclarecer el destino de los desaparecidos y para proteger los derechos del núcleo familiar.

- Garantizar un apoyo jurídico por parte de las autoridades pertinentes, en el caso de que la familia desee emprender un proceso judicial para hacer frente a sus necesidades en materia administrativa o civil (crédito hipotecario, custodia de los hijos, etc.).

\section{Reconocimiento y reparación por parte de las autoridades}

Los familiares consideran que su situación no ha sido suficientemente reconocida por las autoridades. A través del reconocimiento, esperan que estas asuman su responsabilidad en la búsqueda de su ser querido y que les proporcionen información regular sobre las investigaciones.

La mayoría de las familias quisiera tener contacto con asociaciones de familiares de personas desaparecidas $y$, particularmente, para obtener información y apoyo en la búsqueda. En relación con la posibilidad de recibir una asistencia de carácter económico a modo de reparación, los entrevistados consideran que aceptarla implicaría el cese de la búsqueda de su ser querido por parte de las autoridades. Por esta razón, muchos de ellos mencionaron que no aceptarían una ayuda económica como parte de una reparación. Sin embargo, aceptarían un apoyo económico para continuar con la búsqueda.

Más que la sanción de los responsables en relación con la desaparición, las familias de migrantes desaparecidos priorizan la obtención de respuestas por parte de las autoridades acerca de lo ocurrido. Esto puede estar relacionado con la esperanza de encontrar con vida a su ser querido y de que este no haya sido víctima de un delito.

\section{Recomendaciones}

- Reconocer a los familiares de personas desaparecidas como víctimas mediante un estatuto jurídico específico que les permita acceder a los derechos que ayuden a satisfacer sus necesidades.

- Dar a conocer la problemática de los desaparecidos y de sus familiares, mediante la realización de actos públicos y campañas mediáticas, con el objetivo de sensibilizar a las instituciones del Estado y a la población en general sobre su situación, para que se tomen medidas.

- Difundir las medidas que se están adoptando para responder a la problemática. De esta forma no solo se reconocería la situación, sino que además se informaría a la sociedad en su conjunto acerca de la intención de las autoridades de atender el problema. Esto es muy significativo a nivel colectivo, ya que la sociedad en su conjunto también es víctima de la desaparición. 